\title{
Social touch deprivation during COVID-19: effects on psychological wellbeing, tolerating isolation and craving interpersonal touch
}

\author{
Mariana von Mohr ${ }^{1,2+*}$, Louise P. Kirsch ${ }^{3+*}$, Aikaterini Fotopoulou ${ }^{4}$
}

\begin{abstract}
${ }^{1}$ Lab of Action and Body, Department of Psychology, Royal Holloway, University of London, United Kingdom

${ }^{2}$ Departamento de Psicología, Universidad Iberoamericana, México

${ }^{3}$ Institute for Intelligent Systems and Robotics (ISIR), Sorbonne Université, Paris, France

${ }^{4}$ Research Department of Clinical, Educational and Health Psychology, University College London, United Kingdom
\end{abstract}

*Corresponding Authors:

Mariana von Mohr, Department of Psychology, Royal Holloway University of London, Egham TW20 OEX, Email: mariana.vonmohr@rhul.ac.uk

Louise P. Kirsch, Institut des Systèmes Intelligents et de Robotique (ISIR), Sorbonne Université, Paris 75005, Email: kirsch.lou@gmail.com

†Authors share first authorship 


\begin{abstract}
Social touch has positive effects on social affiliation and stress alleviation. However, its ubiquitous presence in human life does not allow the study of social touch deprivation 'in the wild'. Nevertheless, COVID-19-related restrictions such as social distancing allowed the systematic study of the degree to which social distancing affects tactile experiences and mental health. In this study, 1746 participants completed an online-survey to examine intimate, friendly and professional touch experiences during COVID-19-related restrictions, their impact on mental health and the extent to which touch deprivation results in craving touch. We found that intimate touch deprivation during COVID-19-related restrictions is associated with worse psychological wellbeing, even though this type of touch is still the most experienced during the pandemic. Moreover, intimate touch is reported as the type of touch most craved during this period, thus being more prominent as the days practicing social distancing increase. However, our results also show that the degree to which individuals crave touch during this period depends on individual differences in attachment style: the more anxiously-attached, the more touch is craved; with the reverse pattern for avoidantly-attached. These findings point to the important role of interpersonal and particularly intimate touch in times of distress and uncertainty.
\end{abstract}

\title{
Keywords
}

Social touch, COVID-19, anxiety, wellbeing, attachment 


\section{Introduction}

The COVID-19 pandemic presents a unique challenge to societies all over the globe. In order to hinder the accelerating growth of infections, changes in the core social habits of people have become essential. For example, citizens are required to engage in 'physical distancing', initially referred to as 'social distancing' by the World Health Organization (WHO); that is, the minimization of close contacts with others. Notably, the change in term was due because it is important to encourage social interactions (e.g., virtual communications) during epidemic periods. Indeed, social connection and support, even in the form of texts (1), has beneficial effects on distressing events (2) and physical health $(3,4)$. In particular, social supportive behaviours following stress conditions seem to attenuate multiple stress systems, including the autonomic nervous system and hypothalamic-pituitary-adrenal (HPA) axis (4), possibly mediated by neuropeptides involved in social bonding and affiliative behavior, including oxytocin (5). Further, neuroimaging studies indicate that social support reduces activity in brain regions implicated in emotion regulation (i.e., anterior cingulate cortex, dorsolateral and ventrolateral prefrontal cortex) $(6,7)$.

However, a particularly effective form of communicating (non-verbal) support, which in addition facilitates the formation and maintenance of social bonds, is touch (8-10). The potential benefits of touch have been studied in many fields, ranging from animal studies to developmental and adult psychological and neuroscientific studies in humans (11-14). On the one hand, social touch is thought to possess positive hedonic value (although clearly this value depends on the specific context, i.e., touch may not always be welcome or pleasant), in order to promote affiliative and prosocial behaviour (15). For example, the effects of touch in social interactions have been shown to increase the liking of a person (16-18) as well as generosity and compliance (18-20). On the other hand, social touch serves as a form of bonding and reinforcing alliances $(21,22)$. For example, in non-human mammals such as primates, grooming is typically observed within close conspecifics, such as in maternal behaviour, with neurotransmitters involved in social bonding (e.g., oxytocin) mediating such effects (23). In humans, caregiving touch is essential for growth and development in infancy and for wellbeing and bonding in adulthood. Touch actively reduces infant stress by increasing positive affect $(24,25)$ and calms infants in pain and discomfort (14). In the context of attachment theory (26), studies support the facilitating role of touch in establishing the social bond between infant and caregivers (27-29). Touch also has a lifelong effect on human bonding. For instance, a recent study suggests that in romantic couples, selfreports of mutual grooming are positively correlated with relationship quality and previous experiences of familial affection (30).

Moreover, touch by conspecifics has analgesic and stress-alleviating effects (31) mediated by neurobiological pathways involved in social bonding (32). In particular, in humans, social touch has been suggested as a stress buffer, playing a critical regulatory role in the body's responses, 
including cortisol and heart rate responses (33), to acute life stressors, which ultimately promotes social connection (34). For example, a recent study suggests that touching a teddy bear mitigates feelings of social exclusion to increase pro-social behaviour (35). Similarly, studies have shown that slow, gentle social touch, reduces feelings of social exclusion (10). Moreover, functional neuroimaging studies have shown an attenuation of neural responses typically implicated in affective regulation when social embodied support (e.g., hand-holding by a romantic partner) is provided in the face of threat (Coan et al., 2006), including pain $(36,37)$. In line with the notion that we have adapted to the presence and active care of other conspecifics (38-40), with our emotions and sense of selfhood being constituted on the basis of early social interactions, including touch $(41,42)$. However, given physical distancing regulations during the COVID-19 pandemic, our ability to provide and receive this type of support has been likely affected. This stands to be particularly detrimental as the pandemic has signalled a period of global uncertainty, with various mental health consequences such as an increase in loneliness and symptoms of anxiety and depression (43).

Here, participants $(\mathrm{N}=1746)$ were asked to complete an online survey to examine touch experiences during COVID-19, and whether these experiences are associated with individual's psychological wellbeing, including anxiety, feelings of loneliness, mental health and tolerance for isolation. Specifically, we examined whether the amount of touch experienced in the past week (i.e., during COVID-19-related social restrictions) from intimate, friendly or professional sources differently influenced the aforementioned measures of wellbeing. Given literature suggesting that touch is more powerful when provided by a close other $(6,37)$ with its regulatory effects mediated by psychological intimacy (44), we expected that, in particular, the more intimate touch experienced in the past week, the better the wellbeing. By contrast, we expected that the more the lack of touch during COVID-19-related social restrictions (by taking into account touch experienced before COVID-19), the worse the well-being.

Furthermore, we also examined whether the degree to which participants would have wanted to experience intimate, friendly and professional touch in the past week depended on the number of days practicing social distancing, controlling for lack of touch. Given literature suggesting that threats to social connection (e.g., ostracism or isolation) in turn promote seeking social reconnection and proximity $(32,45,46)$, we expected that the more days participants had been practicing social distancing, the more touch they would have wanted to experience in the past week.

In addition, it is known that different people crave touch to different degrees; in fact, some people may not even want to experience it in times of physical or social threat (47). Therefore, we examined whether wanting touch in the past week (i.e., during COVID-19 related lockdown) depended on individual differences, such as (i) adult attachment style and (ii) attitudes and experiences towards touch. Adult attachment style was measured using a well-validated questionnaire (Experiences in close relationships - short ; ECR-S) (48). This questionnaire pertains 
to adult romantic relationships and takes a dimensional approach, yielding continuous scores of attachment anxiety and attachment avoidance. Given that attachment anxiety is characterized by a need for emotional closeness, worries about rejection and abandonment, and over-dependence on others, which seem to extend to touch perception (49), we expected that individuals scoring higher on attachment anxiety would report to have wanted to experience more touch in the past week. By contrast, given that attachment avoidance is characterized by a need for emotional distance, as well as a resistance to trusting and depending on others, we expected that individuals scoring higher on attachment avoidance would report to have wanted less touch in the past week.

With respect to positive attitudes and experiences towards touch, we adapted the Touch Experiences and Attitudes Questionnaire (TEAQ) (50) by selecting an item from each component (e.g., friends and family touch, current intimate touch, childhood touch, etc.). Here we expected that individuals scoring higher on positive attitudes and experiences towards touch, would report wanting more touch. Moreover, taking advantage of the TEAQ different components, we assessed whether individual items predicted wanting touch in the past week. We were particularly interested in the childhood touch component, given that attachment representations are thought to originate in early experiences with primary caregiver(s), and this may extend to touch behaviours $(51,52)$. In this sense, we expected that individuals scoring higher on childhood touch experiences, would report wanting more touch in the past week.

\section{Methods}

\subsection{Participants}

1746 participants were recruited online as part of a larger study on touch during COVID-19related social restrictions. Participants were asked to complete a survey about their tactile experiences during (and before) COVID-19-related restrictions, as well as other self-reported measures about their well-being, including feelings of loneliness (adapted from the UCLA loneliness scale; (53), as suggested by the office for national statistics, 2018), anxiety (STAI-SF ; (54)), mental health and tolerance for isolation. Additional measures such as attitudes and experiences towards touch (adapted from the TEAQ; (50)) and experiences in close relationships (ECR-S,(48)) were also collected.

746 participants were recruited via social media (e.g., Facebook, Twitter) and 1000 were recruited via Prolific (https://prolific.ac/) prescreened for approval rating (i.e. how well participant performed in previous studies) at $>70 \%$ as well as for country of residence (United Kingdom, France and Mexico), as these countries had not yet lifted severe social distancing restrictions at the time of recruitment and were the countries that we had the most prevalence in those recruited via social media. Participants recruited via prolific were paid $£ 2.50$ for their time $(\sim 20$ min). Participants recruited via social media were recruited between the $23 / 04 / 2020$ and the 10/05/2020; participants recruited via prolific were recruited between the 08/05/2020 and 10/05/2020. In these periods, all countries involved were under severe social restrictions (see below) and data collection was finished before countries started to lift lockdown restrictions. Out 
of the 1746 participants, 256 (104 from social media and 152 from prolific) reported having been diagnosed in the past/present with a psychiatric disorder and were thus were removed from data analyses, resulting in a final sample size of 1490 participants (949 female, 539 male, 2 other; Mage $=37.08, \mathrm{SD}_{\text {age }}=14.30$ ).

On average, people reported having been practicing social distancing 46.41 days (SD= 10.56); with the level of regulations applied to their country being advice to not engage in social interactions (i.e., social distancing; $n=71$ ); lockdown (advice to stay at home unless you need to go out, prohibition of social gatherings and interactions, fines in order for those who do not adhere to regulation; $n=808$ ); and complete lockdown (e.g. prohibited to leave the house without a clear purpose, stay in a close radius of your house, most stores and businesses closed; $n=611$ ). In terms of geographical location, 174 reported a country of residence within Latin America (e.g., Mexico, Argentina), 368 within Europe (e.g., France, Spain Greece), 892 in the United Kingdom and 56 outside the aforementioned regions (e.g., Australia, Canada, United States). With respect to household, 187 reported living alone, 438 reported living with one person, 306 with two, 241 with three, 193 with four, 86 with five, and 39 with more than five people.

\subsection{Measures}

\subsubsection{Touch Experience during COVID-19 related lockdown period}

Using a visual analogue scale ranging from 0 'not at all' to 100 'a lot' for three items corresponding to different types of 'social' touch, (i.e., (a) intimate, e.g., kiss, hugs, caress from partner or close family; (b) friendly, e.g., hugs and high-fives from friends or acquaintances; (c) professional, e.g. handshakes, tap on the shoulder from colleagues, touch from carers), participants were asked to answer the following questions about their tactile experience: (i) "Before COVID-19, How much touch of these different 'social' types of touch were you getting?"; (ii) "In the past week, How much touch of these different types of 'social' touch have you been getting?"; (iii) "In the past week, How much would you have wanted to experience these different types of 'social' touch?". Please note that the last two questions relate to participant's amount of touch experienced and wanted, respectively, during a period of COVID19-related restrictions. To obtain an index of lack of touch during this period we subtracted the scores from question (ii) touch experienced in the past week from question (i) touch experienced before COVID19, separately for each item (i.e., intimate, friendly, professional). See Figure 1 for plots with means and error bars.

\subsubsection{Anxiety}

To examine anxiety, we used the well-validated six-item short-form of the state scale of the StateTrait Anxiety Inventory (STAI-SF; (54)). It comprises six items rated on a 4-point scale (1= not at all and $4=$ very much). Items were summed (after reverse-scoring appropriate items) and multiplied by $20 / 6$ to obtain an anxiety score. The STAI-SF is well-validated and demonstrates very good internal consistency (Cronbach's $\alpha=0.84)$. On average, anxiety score was $M=44.09(S D=13.65)$. Higher scores denote more anxiety.

\subsubsection{Loneliness}


To examine feelings of loneliness, we used three items from the UCLA loneliness scale (53) as an implicit measure of loneliness, as well as an explicit item asking 'How often do you feel lonely?', as recommended by the office for national statistics (2018). Items were rated on a 4-point ( $0=\mathrm{Never}$ and $3=0$ ften) and a 5-point scale (1=Never and 5=Often/Always), for implicit and explicit items, respectively. A summed score was computed for our implicit measure of loneliness based on the three UCLA items, and demonstrated good internal consistency with the explicit measure of loneliness, Cronbach's $\alpha=0.79$. Thus, we averaged implicit and explicit loneliness scores to produce an index of loneliness for each participant, with higher scores denoting more perceived loneliness. On average, loneliness score was $\mathrm{M}=3.61$ (SD=1.62).

\subsubsection{Tolerance to isolation}

To examine the degree to which participants were tolerating isolation, participants were asked to answer the following question: "How much are you tolerating isolation (emotionally)?" using a visual analogue scale ranging from 0 'Not at all' to 100 'extremely'. On average, tolerance to isolation score was $\mathrm{M}=66.37$ ( $\mathrm{SD}=24.99)$.

\subsubsection{Mental Health}

As an index of mental health, participants were asked to judge their mental health over the past week using a five-point scale ( $1=$ very poor and $5=$ very good). On average, mental health score was $\mathrm{M}=3.55$ (SD=.95).

\subsubsection{Self-report measure of adult attachment style (Experiences in Close Relationships Short - ECR-S)}

As an index of adult attachment style we collected The ECR-S (48) on the participants recruited from Prolific $(n=1000)$. The ECR-S comprises 12 items rated on a 7-point scale (1=strongly disagree and $7=$ strongly agree) regarding the general experience of intimate adult relationships; 6 items pertain to attachment anxiety and 6 to attachment avoidance. Item responses are averaged (after reverse-scoring appropriate items) separately for each subscale to produce a mean score for attachment anxiety and attachment avoidance, with higher scores denoting greater attachment insecurity. The ECR-S is well-validated (Wei et al., 2007) and demonstrates good internal consistency: Cronbach's $\alpha=.75$ for attachment anxiety and Cronbach's $\alpha=.80$ for attachment avoidance. On average, attachment anxiety score was $M=3.72 \quad(S D=1.18)$ and attachment avoidance $\mathrm{M}=2.67$ (SD=1.09).

\subsubsection{Attitudes and Experiences towards touch}

To examine attitudes and experiences towards touch, we used 7 items rated on a 5-point scale (1 $=$ Disagree strongly and $7=$ Agree strongly) from the Touch Experiences and Attitudes Questionnaire (TEAQ; 49). Each item was selected as they correspond to one of the six components from the TEAQ, namely: friends and family touch (FFT), current intimate touch (CIT), childhood touch (ChT), attitude to self-care (ASC), attitude to intimate touch (AIT) and attitude to unfamiliar touch (AUT). However, we included one more item from the ChT component, to 
examine the effects of childhood touch further. After reverse-scoring appropriate items, items demonstrated moderate internal consistency, Cronbach's $\alpha=0.63$. Items were summed to produce a mean score for attitudes and experiences towards touch, with higher scores denoting more positive attitudes and experiences. On average, touch attitudes and experiences score was $M=24.31$ (SD=5.27).

\subsubsection{Other measures}

Participants answered other questions, such as demographics (age, country of residence, nationality), and COVID-19 related questions (to what degree participants were practicing social distancing, days in lockdown, infection by COVID-19). These variables were taken into account as control measures where needed.

Correlations between variables of interest are summarized in Supplementary Figure S3.

\subsection{Statistical analyses}

All analyses were carried out in Stata 15. For ease of reference, details on each analysis conducted are presented with the corresponding results below. Given the big sample, we opted for a conservative approach of $p<.01$ rather than the conventional $p<.05$ to denote statistical significance.

\section{Results}

\subsection{Descriptive statistics and preliminary analyses}

\subsubsection{Touch Experience}

A repeated measures ANOVA, specifying within-subjects factors of type of touch (intimate, friendly, professional), was conducted separately on each measure of touch experience: touch experienced before COVID-19, touch experienced in the past week (during COVID-19-related social restrictions), lack of touch (as the difference between before COVID-19 and past week), and wanting touch (during COVID-19-related social restrictions). The main effect of type of touch was statistically significant across all (all $F^{\prime} s>340, p^{\prime} s<.000, \eta_{p}{ }^{2} s>.19$ ). Notably, whilst intimate (vs. friendly and vs. professional) touch was self-reported as the most experienced (both before and during COVID-19) and wanted touch, $p^{\prime} s<.000$, friendly touch was the most lacked during COVID19-related social restrictions, $p^{\prime} s<.000$; See Figure 1 . Note that same pattern of effects remains when applying Bonferroni correction. 
A. Touch experienced before COVID-19

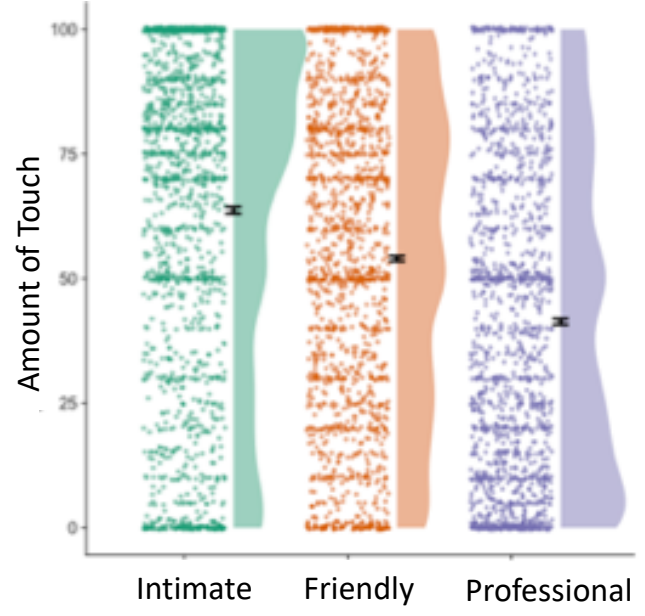

\section{Lack of touch}

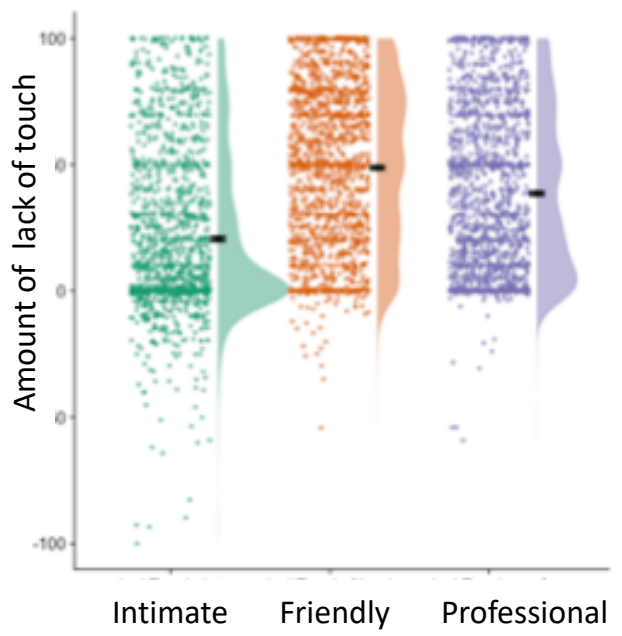

\section{B. Touch experienced in the past week}

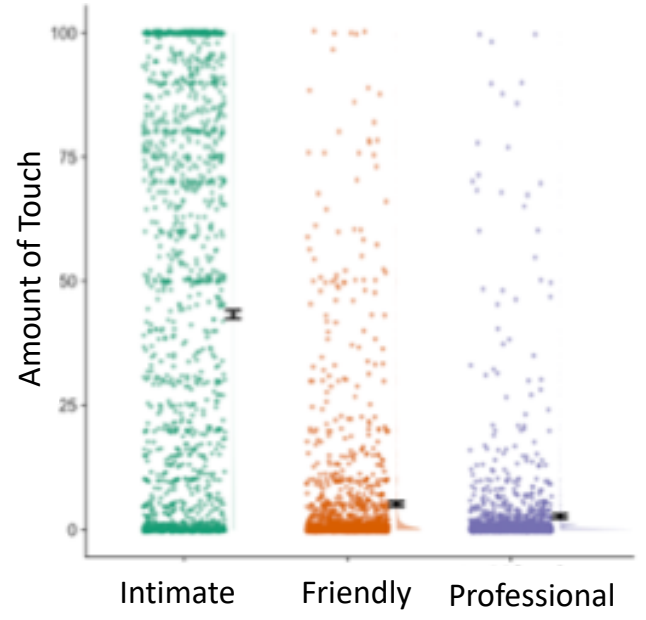

D. Wanting to experience touch in the past week

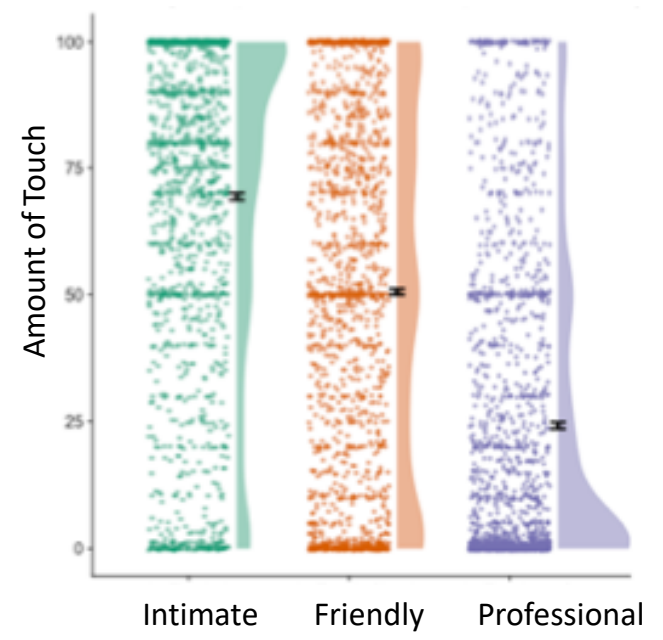

Figure 1. Ratings for touch experienced during COVID-19 for the three types of social touch: intimate, professional and friendly. (A) “Before COVID-19, How much touch of these different 'social' types of touch were you getting?”; (B) “In the past week, How much touch of these different types of 'social' touch have you been getting?"; (C) Computed score for lack of touch during COVID-19; touch experienced in the last week was subtracted from touch experienced before COVID-19; (D) "In the past week, How much would you have wanted to experience these different types of 'social' touch?". Group distributions as un-mirrored violin plots (probability density functions), individual data points, mean and error bars denoting $+/-1$ SEM.

\subsubsection{Has practicing social distancing resulted in a lack of touch?}

Pearson correlations indicated a significantly weak positive correlation between practicing social distancing and lack of touch for friendly, $r=.09, p=.000$, but not intimate, $r=.02, p=.515$, professional, $r=.01, p=.663$, or their average, $r=.05, p=.041$. Note that the same pattern of effects remains for friendly touch when using Bonferroni adjusted alpha levels of .0125 per test (0.05/4). See Figure 2A.

\subsubsection{Does the lack of touch, results in people wanting more touch?}

Pearson correlations indicated a significant positive correlation between wanting touch in the past week and lack of touch, weak for intimate, $r=.09, p=.001$, but strong for friendly, $r=.48, p=.000$, 
professional, $r=.46, p=.000$, and their average, $r=.43, p=.000$. Note that the same pattern of effects remains when using Bonferroni adjusted alpha levels of .0125 per test (0.05/4). See Figure $2 B$.

(A)

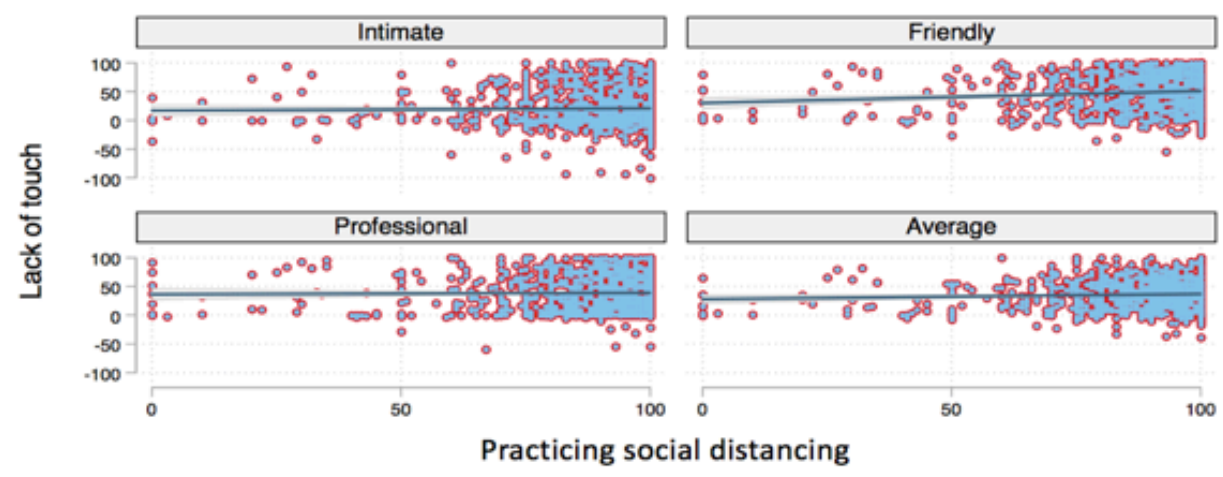

(B)

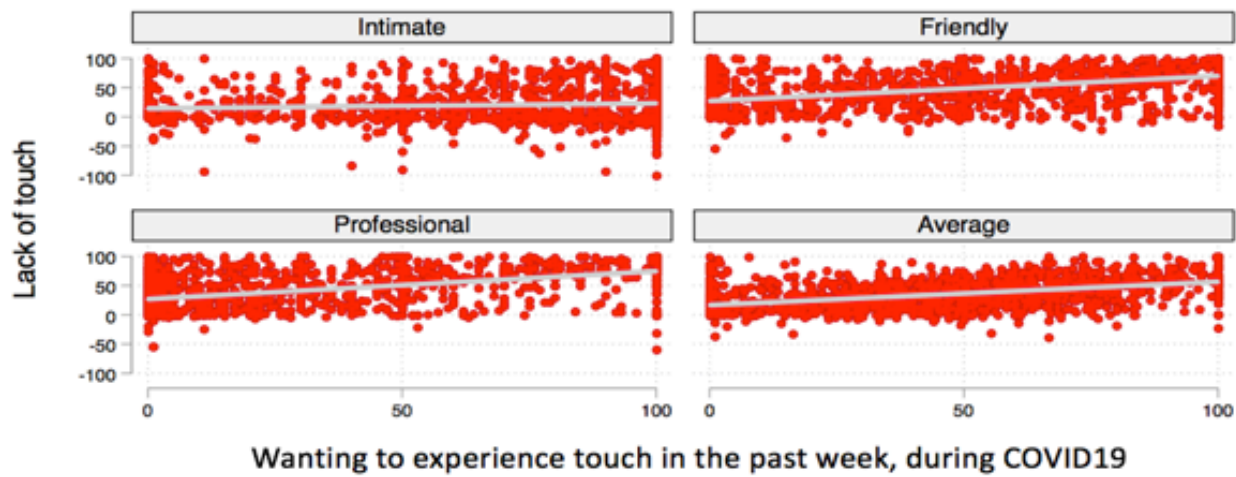

Figure 2. (A) Relationship between computed lack of touch (touch experienced before COVID-19 minus touch experienced in the past week) and the degree to which participants reported practicing social distancing (visual analogue scale ranging from 0 'not at all' to 100 'extremely') for intimate, friendly and professional touch, as well as their average. (B) Relationship between computed lack of touch and the amount of touch that participants would have wanted to experience in the past week for intimate, friendly and professional touch, as well as their average.

\subsection{Main analyses}

\subsubsection{Does the amount of touch we get during COVID-19 influence our well-being and ability to tolerate social isolation?}

To examine whether experiencing different types of social touch (i.e., professional, friendly and intimate) influences our wellbeing, we conducted multiple regressions separately on the following outcome variables: anxiety, loneliness, tolerance to isolation and mental health scores. Intimate, friendly and professional touch ratings in response to the question "In the past week, how much touch of these different types of 'social' touch have you been getting?" were entered as continuous predictors. Continuous variables were mean-centered to avoid multicollinearity issues (55).

Given that the above effects may not be specific to the amount of touch experienced during COVID-19, we conducted the same multiple regressions as described above but this time we entered our computed scores of lack of touch (i.e., touch experienced in the past week subtracted from the that experienced before COVDID19) in response to intimate, friendly and 
professional touch as predictor variables. We expected the same pattern of effects as above, yet in the opposite direction.

When applicable, we specified bootstrap with 1000 repetitions and seed of 1 to account for non-normality of our data; with these analyses yielding the same pattern of results.

3.2.1.1 Anxiety. A significant regression equation was found, $F(3,1486)=7.61, p<.000$, with an $\mathrm{R}^{2}$ of .02 . Intimate, $\mathrm{b}=-.04, \mathrm{SE}=.01, \mathrm{p}<.000$, eta ${ }^{2}=.012$, but not friendly, $\mathrm{b}=-.04, \mathrm{SE}=.03, \mathrm{p}=.142$, eta ${ }^{2}=.001$, or professional touch, $b=.08, \mathrm{SE}=.04, \mathrm{p}=.048$, eta ${ }^{2}=.003$, was a significant predictor of anxiety. In sum, the more intimate touch experienced in the past week, the less the anxiety. Critically, the same pattern of results, yet in opposite direction, were observed when entering lack of touch computed scores instead. Namely, the more the lack of intimate touch, $b=.04, \mathrm{SE}=.01$, $p<.001$, eta ${ }^{2}=.009$ (but not friendly or professional, $p^{\prime} s>.083$ ), the more participants' anxiety level was high; see Figure 3.

3.2.1.2 Loneliness. A significant regression equation was found, $F(3,1486)=47.08, p<.000$, with an $\mathrm{R}^{2}$ of .09 . Intimate, $\mathrm{b}=-.01, \mathrm{SE}=.00, \mathrm{p}<.000$, et ${ }^{2}=.085$ but not friendly, $\mathrm{b}=-.00, \mathrm{SE}=.00$, $p=.309$, eta ${ }^{2}=.0006$ or professional, $b=.00, S E=.00 p=.370$, eta ${ }^{2}=.0005$ touch was a significant predictor of loneliness. In sum, the more intimate touch experienced in the past week, the less the loneliness. Critically the same pattern of results, yet in opposite direction, were observed when entering lack of touch computed scores instead. Namely, the more the lack of intimate touch, $\mathrm{b}=.01, \mathrm{SE}=.00, \mathrm{p}<.001$, eta ${ }^{2}=.02$ (but not friendly or professional, p's $>.236$ ), the more the loneliness; see Figure 3.

3.2.1.3 Mental health. A significant regression equation was found, $F(3,1486)=11.14$, $p<.000$, with an $R^{2}$ of .02 . Intimate, $b=.01, S E=.00, p<.000$, eta ${ }^{2}=.02$, but not friendly, $b=.00, S E=.00$, $p=.958$, eta ${ }^{2}<.000$, or professional touch, $b=.002, S E=.00 p=.385$, eta ${ }^{2}=.0005$, was a significant predictor of mental health. In sum, the more intimate touch experienced in the past week, the better the mental health over the past week. Critically we observe similar pattern of results, yet in opposite direction, when entering lack of touch computed. Namely, the more the lack of intimate touch, $b=-.01, S E=.00, p=.000$, eta ${ }^{2}=.01$ and the less the lack of professional touch, $b=.01, \mathrm{SE}=.00$, $p=.001$, eta ${ }^{2}=.008$ (but not friendly, $p>.236$ ), the worse the mental health; see Figure 3.

3.2.1.4 Tolerance to isolation. A significant regression equation was found, $F(3$, $1486)=8.91, p<.000$, with an $R^{2}$ of .02 . Intimate, $b=.06, S E=.02, p<.000$, eta ${ }^{2}=.009$, but not friendly, $b=-.13, S E=.05, p=.013$, eta ${ }^{2}=.004$ or professional touch, $b=-.08, S E=.08 p=.259$, eta ${ }^{2}=.0009$ was a significant predictor of tolerance for isolation. In sum, the more intimate touch experienced in the past week, the more the tolerance for isolation. Critically we observe the same pattern of results, yet in opposite direction, when entering lack of touch computed scores instead. Namely, the more the lack of intimate touch, $b=-.05, S E=.02, p=.023$, eta ${ }^{2}=.003$ (but not friendly or professional, $p^{\prime} s>$.236), the less the tolerance for isolation; however, the latter did not reach significance at our established alpha levels of .01; see Figure 3. 


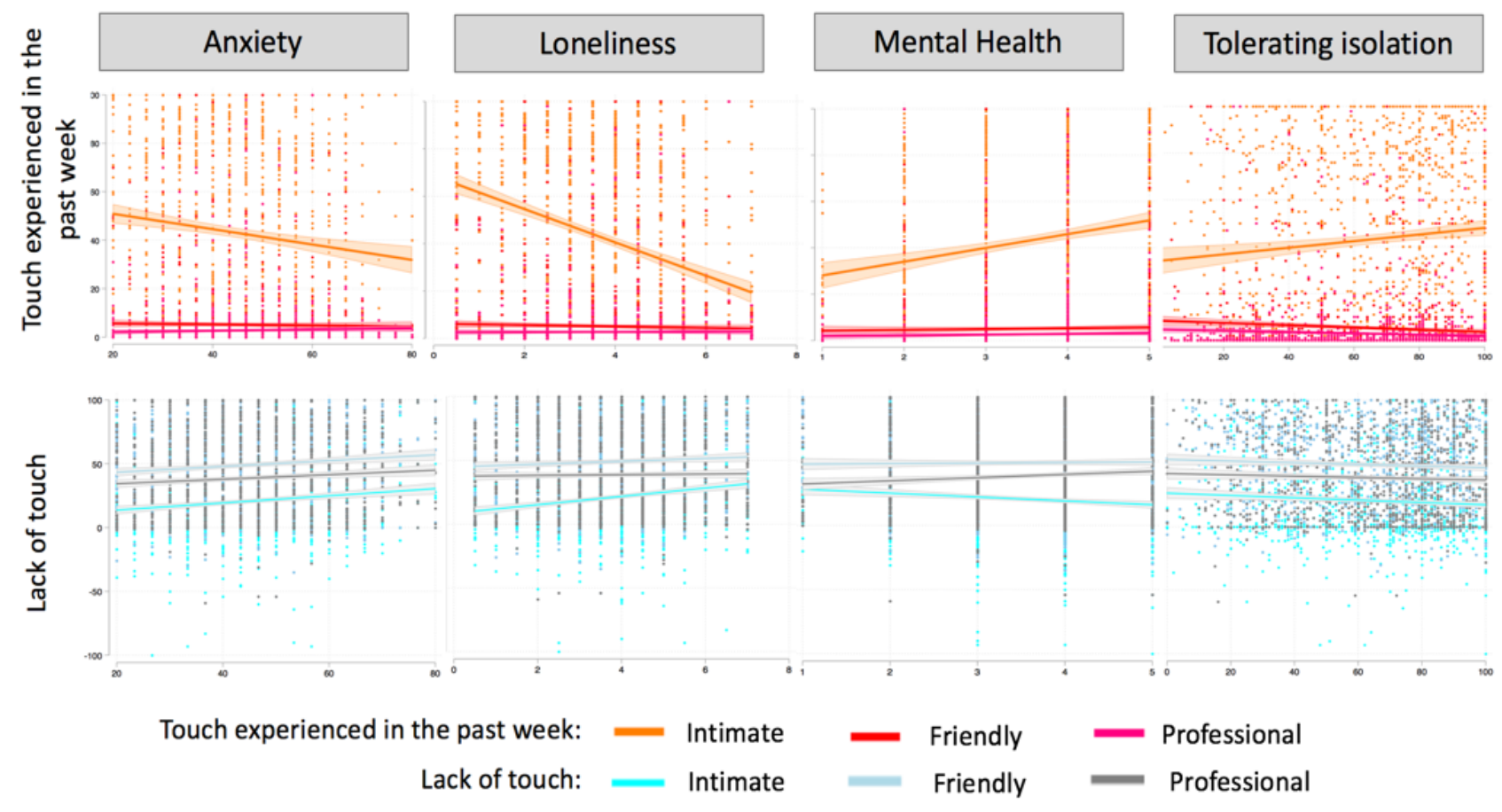

Figure 3. Top panel: Ratings in response to touch experienced in the past week (during COVID-19 related lockdown) in relation to anxiety, loneliness, mental health and tolerating isolation. Bottom panel: computed lack of touch (touch experienced before COVID-19 minus touch experienced in the past week) in relation to anxiety, loneliness, mental health and tolerating isolation.

\subsubsection{What type of touch do people want to experience during COVID-19 related lockdown? And does this depend on days practicing social distance?}

To examine whether wanting different types of touch in the past week was associated with days practicing social distance, we specified a multilevel regression model with wanting touch ratings as the outcome variable and type of touch (intimate, friendly, professional), days practicing social distancing, and their interaction, as predictors variables, and controlled for lack of touch. Days practicing social distancing (continuous variable) was mean-centred in order to avoid multicollinearity issues (55). A random effect was included to account for the repeated assessment of the outcome variable within individual.

The type of touch predicted wanting touch ratings: intimate touch $(M=73.70, S D=.83)$ was rated as more wanted than friendly $(M=46.95, S D=.83)$ and professional $(M=23.47, S D=.81)$ touch (see supplementary materials table S1 for full model results). Days in lockdown also predicted wanting touch, $b=.20, S E=.08, p=.009$. Interestingly, the type of touch by days in lockdown interaction was also significant: friendly, $b=-.24, \mathrm{SE}=.08, \mathrm{p}=.004$, and professional, $b=-.34, \mathrm{SE}=.08$, $p=.000$, touch by days in lockdown was significantly different than intimate touch (base level). Specifically, the more the days in lockdown the more intimate touch people would want to experience; see Figure 4. Note that the effect size for the full model is relatively high $\left(f^{2}=1.06\right)$. 


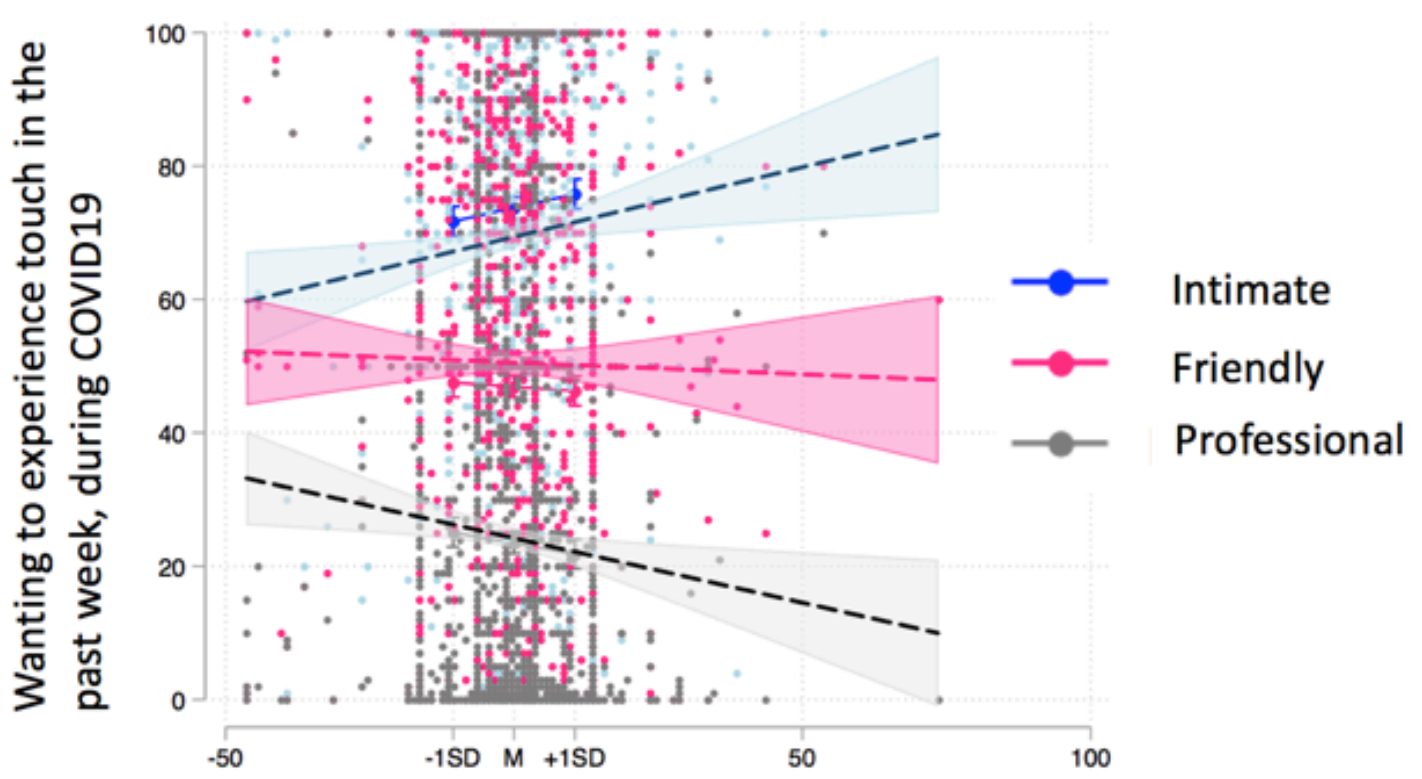

Days practicing social distancing

Figure 4. Interaction between type of touch and days practicing social distancing. Follow-up continuous by categorical interactions were plotted using the 'margins' command at -1SD, mean and +1SD of continuous variables, in this case practicing social distancing. Days practicing social distancing was mean-centred, ranged from 0 to 120 days.

\subsubsection{Does wanting (types of) touch during COVID-19 depend on individual differences?}

\subsubsection{Adult attachment style}

To examine whether wanting different types of touch in the past week was associated with adult attachment style, we specified a multilevel regression model with wanting touch ratings as the outcome variable and type of touch (intimate, friendly, professional), and attachment anxiety, attachment avoidance, as well as their interaction terms, as predictor variables and controlled for lack of touch. Continuous variables were mean-centered in order to avoid multicollinearity issues. A random effect was included to account for the repeated assessment of the outcome variable within individual.

As above, type of touch predicted wanting touch ratings: intimate touch ( $M=70.98$, $S D=1.02)$ was rated as more wanted than friendly $(M=43.28, S D=1.02)$ and professional touch $(\mathrm{M}=20.27, \mathrm{SD}=1.00)$. Attachment anxiety, $\mathrm{b}=3.07, \mathrm{SE}=.86, \mathrm{p}=.000$, and attachment avoidance, $\mathrm{b}=-$ 13.61, $\mathrm{SE}=.94, \mathrm{p}=.000$, predicted wanting touch: the more the attachment anxiety, the more the wanting to experience touch the past week; by contrast, the more the attachment avoidance the less the wanting to experience touch. Moreover, the type of touch by attachment anxiety interaction was significant: friendly, $b=3.13, \mathrm{SE}=.96, \mathrm{p}=.001$, but not professional, $b=-.96, \mathrm{SE}=.96$, $p=.315$, touch by attachment anxiety was significantly different than intimate touch (base level). Specifically, the more the attachment anxiety, the more friendly touch people want. The type of touch by attachment avoidance interaction was also significant: intimate touch by attachment avoidance was significantly different than friendly, $b=8.76, \mathrm{SE}=1.05, \mathrm{p}=.000$, or professional touch, $b=11.71, S E=1.04, p=.000$. Specifically, the more the attachment avoidance, the less intimate touch people want; see Figure 5. In addition, there was a significant attachment avoidance by attachment anxiety interaction, $b=2.15, S E=75, p=.004$ (see supplementary materials Figure S1). 
The type of touch by attachment avoidance by attachment anxiety interaction was non-significant; see supplementary materials Table S2 for full model results. Note that the effect size for the full model is relatively high $\left(\mathrm{f}^{2}=1.21\right)$.
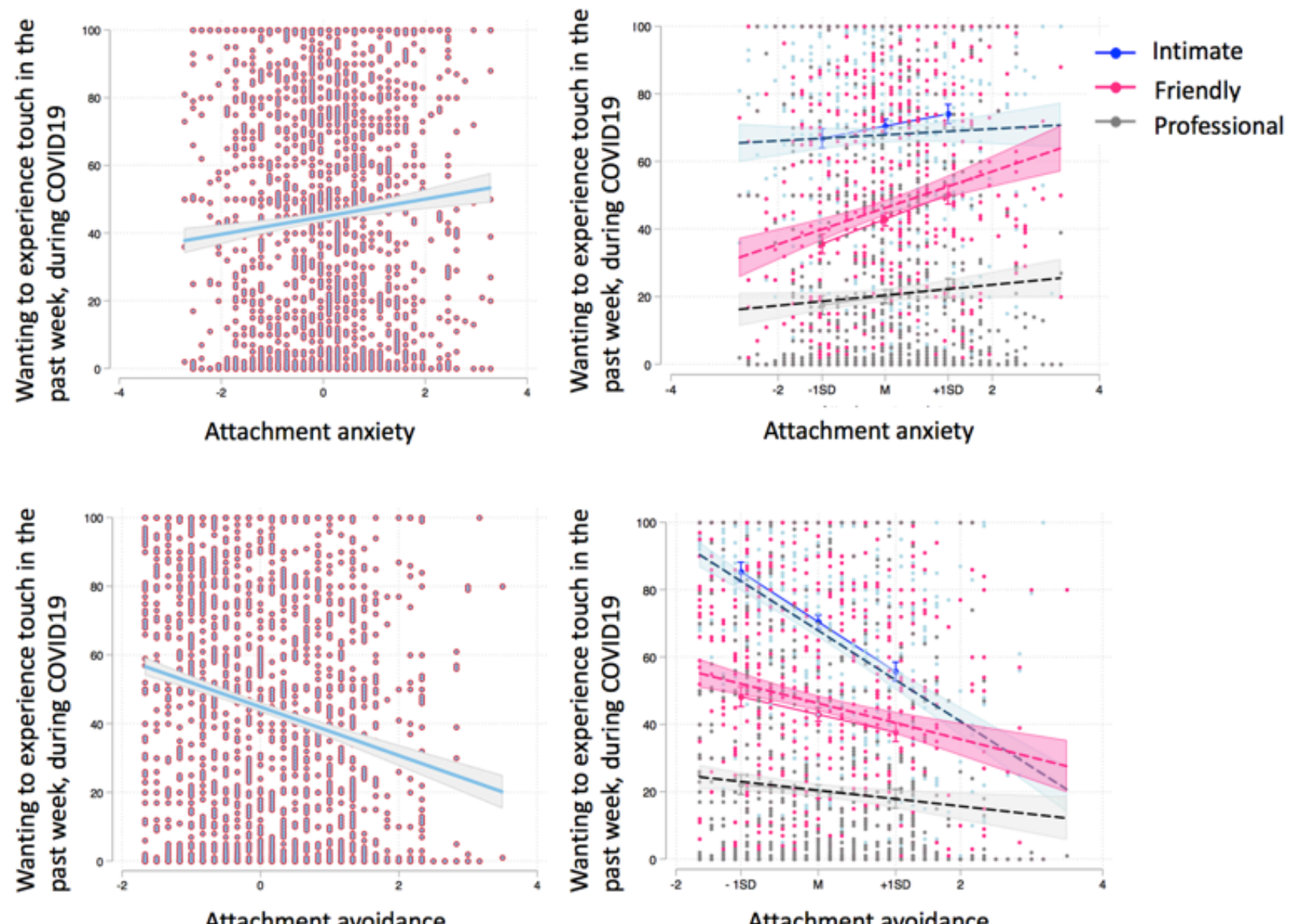

Attachment avoidance

Figure 5. Top panel: Effects of attachment anxiety on wanting touch (left); Interaction between types of touch and attachment anxiety (right) Bottom panel: Effects of attachment avoidance on wanting touch (left). Interaction between types of touch and attachment avoidance (right). Follow-up continuous by categorical (type of touch: intimate, friendly, professional) interactions were plotted using the 'margins' command at $-1 S D$, mean and +1SD of continuous variables.

\subsubsection{Attitudes and experiences towards touch}

To examine whether wanting types of touch in the past week was associated with adult attitudes and experiences towards touch, we specified a multilevel regression model with wanting touch ratings as the outcome variable and type of touch (intimate, friendly, professional), and touch attitudes and experiences scores, as well as their interaction terms, as predictor variables and again controlled for lack of touch. A random effect was included to account for the repeated assessment of the outcome variable within individual.

As above, type of touch predicted wanting touch ratings: intimate touch ( $M=73.30, S D=$ .80) was rated as more wanted than friendly $(M=47.24, S D=.79)$ and professional $(M=23.53$, $\mathrm{SD}=.77$ ) touch. Touch experiences and attitudes predicted wanting touch, $b=2.08, \mathrm{SE}=.15, \mathrm{P}=.000$, indicating that the more positive the touch experiences and attitudes, the more people would have wanted touch the past week. Importantly, the type of touch by touch experiences and 
attitudes interaction was significant: intimate touch by touch experiences and attitudes was significantly different than professional, $b=-1.13, \mathrm{SE}=.17, \mathrm{p}=.000$, but not friendly touch, $b=-.19$, $\mathrm{SE}=.17, \mathrm{p}=.243$. Specifically, the more positive the touch attitudes and experiences, the more intimate and friendly touch people would want to experience; see Figure 6A and supplementary materials for full model results. Note that the effect size for the full model is relatively high $\left(f^{2}=\right.$ 1.03).

To further examine whether wanting touch was associated with specific components of the attitudes and experiences towards touch measure, we specified a multilevel regression model with wanting touch ratings as the outcome variable and included the seven items of the experiences and attitudes towards touch measure as predictor variables. Interestingly, all items predicted wanting touch, $p^{\prime} s<.001$, except for the two items corresponding to the childhood touch experience (ChT) component, p's> .195, namely: “My parents were not very physically affectionate towards me during my childhood" and "As a child my parents would tuck me up in bed every night and give me a hug and a kiss goodnight". See supplementary materials Table S4 for full model results.

(A)

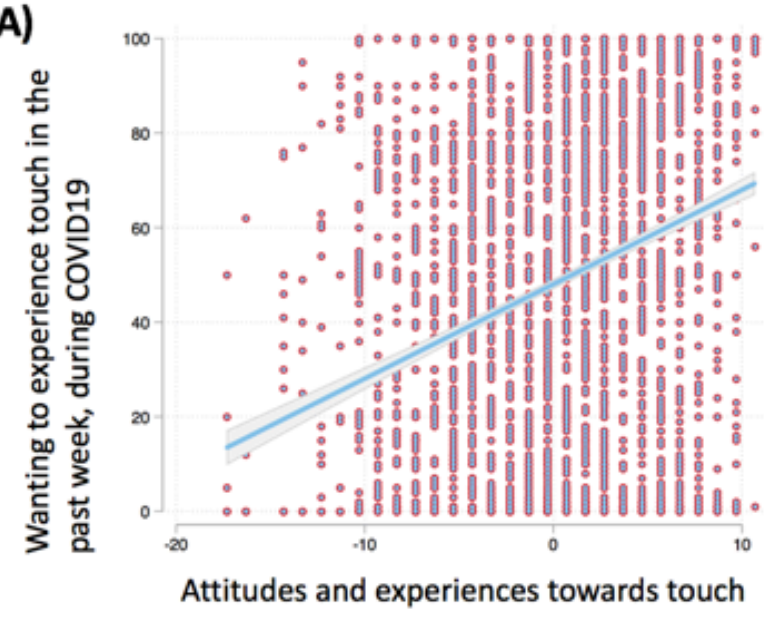

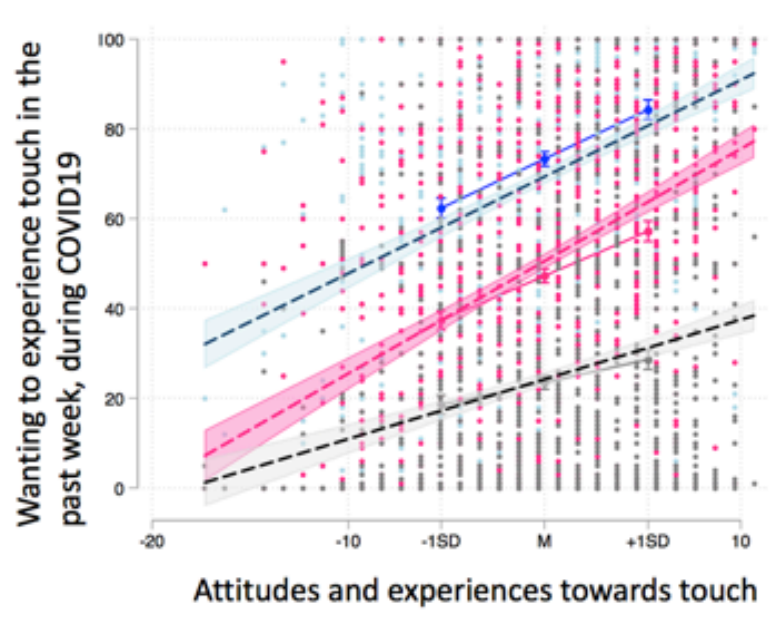

Figure 6. (A) Left panel: Effects of attitudes and experiences towards touch on wanting to experience touch. Right panel: Interaction between types of touch and wanting to experience touch.

\section{Discussion}

COVID-19-related restrictions inevitably affected core social habits of citizens, including tactile behaviours (with our data supporting this notion; see Figure 1 and Figure 2). Given growing lab and epidemiological evidence suggesting that social touch has beneficial effects on well-being $(31,33)$, the present study first investigated whether the touch deprivation caused by COVID-19related restrictions was associated with worse psychological outcomes. We found that the more the intimate touch (but not friendly or professional) experienced in the past week (i.e., during COVID-19), the better the targeted psychological outcomes: self-reported anxiety, feelings of loneliness, general mental health state and perceived tolerance for isolation (emotionally). These findings are consistent with growing evidence suggesting that the beneficial effects of touch are 
context-specific $(6,37)$. Indeed, touch is central to intimate, romantic relationships $(56)$, and the regulatory role of touch seems to be mediated by psychological intimacy (44). These findings are important given that anxiety, depression and stress have been shown to be common reactions to the COVID-19 pandemic (43) and intimate touch may work as a protective factor. Moreover, we also found that the more the lack of intimate touch (but not friendly or professional), the worse the psychological outcomes (except for tolerance to isolation, which was non-significant at our predetermined alpha level of .01). Interestingly, intimate (vs. friendly and professional) touch was reported as the least deprived type of touch (see Figure 1); yet such effects seem to be the most pervasive on psychological well-being. Taken together, these findings suggest that experiencing intimate touch (e.g., kiss, hugs, caress from partner or close family) plays an important role on our well-being during COVID-19. Future studies should examine in finer detail whether these effects are driven by the precise nature of the touch relation, e.g. close family member vs. partner, or by the type of touch itself, e.g. kisses versus hugs, or both (for example by using the longing for interpersonal touch picture questionnaire (57)).

Furthermore, we found that the more days practicing COVID-19-related social distancing, the more individuals wanted to experience touch. Consistent with literature suggesting that threats to social connection promote seeking of social re-connection and proximity $(32,45,58)$ we hypothesized that the more people perceive threat to their social connections (which likely increases by time practicing social distancing), the more it increases their craving/wanting of touch. In line with this thinking, however, we expected friendly touch in particular to increase as a function of days practicing social distancing (as it is the type of touch that was found to lack the most during COVID-19; see Figure 1). Nevertheless, we found that the more the days practicing social distancing, the more individuals wanted to experience intimate touch (note that we controlled for lack of touch in our analyses). One possible explanation is that as days practicing social distancing increase, the less individuals seek or want friendly and professional touch as these sources of touch are less available to them (there was in fact a negative association between the amount of touch experienced in the past week and days practicing social distancing, for friendly and professional touch only, p's<.001.) and in turn crave intimate touch during the pandemic, as they have continued to experience it, relatively to a higher degree. Indeed, touch has context-specific rewarding properties, which are reinforced by experience (59).

Critically, we also found that the degree of wanting to experience touch in the past week (i.e., during COVID-19 related lockdown) further depended on individual differences in adult attachment style as well as attitudes and experiences towards touch. Specifically, the higher the attachment anxiety, the more individuals would have wanted to experience touch in the past week, and particularly, friendly touch. Given that anxious attachment is associated with craving closeness and reassurance from others (60), we hypothesize that particularly in a time when physical proximity with others is impaired, they would need and want more tactile support. The fact that anxious attachment is also characterized by worries about rejection and abandonment from close others (60), might in turn make them want more friendly rather than intimate touch. 
By contrast we found that the higher the attachment avoidance, the less individuals would have wanted to experience touch in the past week, and particularly, intimate touch. Given that avoidant attachment is associated with a need for emotional distance and reduced proximity seeking $(61,62)$, we hypothesize that they would want to experience less touch during COVID-19, particularly from intimate, close others. This finding is consistent with research suggesting that avoidant individuals prefer to cope with pain on their own. For example, a recent study suggests that embodied support from a romantic partner results in more pain (63), which may extend to touch. However, the available measures do not allow us to know if this pattern of wanting touch depending on attachment style is specific to COVID-19. It is indeed possible that this is a trait characteristic (i.e., present before COVID-19) and future studies should examine this issue.

With respect to attitudes and experiences towards touch, we found that the more the positive attitudes and experiences towards touch, the more individuals would have wanted to experience touch in the past week (during COVID-19). This makes sense as the more positive people feel about touch, the more it might increase its wanting in times of deprivation. Notably, this was particularly the case for intimate and friendly touch, in line with the fact that these are the most frequently experienced types of touch with a positive associated meaning (see (59) for the role of context and motivation on touch). Surprisingly, we found that all items selected from the TEAQ (50) components predicted wanting touch in the past week, except for the Childhood Touch (ChT) items. This was unexpected because tactile exposure seems to relate to tactile, enjoyable experiences with close, familiar others (64), which may be related to attachment and early tactile experiences (49). However, it's worthwhile noting that in contrary to other measures (e.g., adult attachment interview), the ECR-R targets explicit adult romantic relationships, without focusing on childhood experiences with caregivers. As such, even though attachment representations are thought to remain relatively stable across the lifespan (65) it is possible that these may not relate, at least regarding tactile experiences (although see 48).

Our findings should be considered in light of their limitations and directions for future research. First, the current study was based solely on self-report measures and as such, there might be social desirability effects. For example, it is possible that participants reported more touch deprivation than they were actually experiencing, merely because they were primed given the aim of the study. Relatedly, participants could have indicated a similar pattern for wanting touch in the past week to the amount of touch experienced before COVID-19 simply because it was prompted given the order of the questions. Future research should examine this matter by randomizing or counter-balancing these items. Second, some questionnaires (i.e., TEAQ and UCLA loneliness scale) were adapted for this study and not all items were administered. This was done given time constraints. Thus, even though chosen items were selected carefully and in line with recommendation guidelines, future studies should examine whether the present effects remain using the full questionnaires. Third, future research should examine if the current effects depend on demographic variables, such as geographical location, age and gender. We did not examine this issue further as it was beyond the scope of this paper. However, we did examine whether these 
factors played a role on experiencing touch, with results suggesting that geographical location, age, gender, or their interaction, do not play a role on experiencing touch during or before COVID19 ( $p$ 's>.016). Finally, touch experience during COVID-19 in clinical samples, such as patients diagnosed with anxiety or depression, remains to be fully examined. In our sample, out of the 256 participants excluded for reporting a diagnosed psychiatric condition: 54 reported anxiety, 55 depression, 41 reported both, and 106 other psychiatric condition. While preliminary analyses indicate that there is no evidence to suggest that wanting touch during COVID-19 depends on these psychiatric categories; we found that participants reporting other psychiatric condition, as well as those reporting anxiety and depression, had the lowest scores in lacking touch during COVID-19. However, the main effect of anxiety and depression did not reach significance at our predetermined $p$ value of .01 (see Supplementary Materials). Future research is still needed to examine this issue further in actual clinical samples, with the adequate tools to assess psychiatric conditions, before drawing any firm conclusions.

In sum, these findings corroborate and extend previous literature on the important role of touch, particularly by close, intimate others, in times of social distancing, psychological distress and social pain. We show that intimate touch deprivation during COVID-19 is associated with worse psychological wellbeing. In addition to these effects on wellbeing, individuals seem to crave this type of intimate touch the most during COVID-19, with such effects being more prominent the more the days they have been practicing social distancing. However, craving touch during COVID19 depends on individual differences in attachment style as well as in attitudes and experiences towards touch.

\section{Acknowledgments}

We would like to thank Alkistis Mavrogalou Foti and Ilia Galouzidi for the Greek translation, to Elena Salvadé for the Italian translation, to Paulo Silva for the Portuguese translation, and to Kohinoor Darda for the translation in Marathi. We thank also all the participants that took part and people who shared the survey helping us to gather all this data.

\section{Ethical Statement}

The study was approved by the Ethics Committee at University College London. All participants gave informed consent, and all collected data was anonymous.

\section{Funding Statement}

This work was supported by a European Research Council Consolidator Award [ERC-2018-COG818070] (to AF). 


\section{Data Accessibility}

The data that support the findings of this study are available on the Open Science Framework (https://osf.io/b46cs/).

\section{Competing Interests}

The authors report no competing interests.

\section{Authors' Contributions}

M.V. and L.P.K. had the original idea for this study, and developed the study concept together with A.F. All authors contributed to the final study design. M.V. and L.P.K performed the data analysis and interpretation with additional input A.F. M.V. drafted the manuscript, and all other authors provided critical revisions. All authors approved the final version of the manuscript for submission.

\section{References}

1. Onoda K, Okamoto Y, Nakashima K, Nittono H, Ura M, Yamawaki S. Decreased ventral anterior cingulate cortex activity is associated with reduced social pain during emotional support. Soc Neurosci. 2009;4(5):443-54.

2. Park KO, Wilson MG, Myung SL. Effects of social support at work on depression and organizational productivity. Am J Health Behav. 2004;28(5):444-55.

3. Uchino BN. Social support and health: A review of physiological processes potentially underlying links to disease outcomes. Vol. 29, Journal of Behavioral Medicine. 2006. p. 37787.

4. Ditzen B, Heinrichs M. Psychobiology of social support: The social dimension of stress buffering. Restor Neurol Neurosci. 2014;32(1):149-62.

5. Heinrichs M, Baumgartner T, Kirschbaum C, Ehlert U. Social support and oxytocin interact to suppress cortisol and subjective responses to psychosocial stress. Biol Psychiatry. 2003;54(12):1389-98.

6. Coan J a, Schaefer HS, Davidson RJ. Lending a Hand of the Neural Response to Threat. Psychol Sci. 2006;17(12):1032-9.

7. Eisenberger NI, Taylor SE, Gable SL, Hilmert CJ, Lieberman MD. Neural pathways link social support to attenuated neuroendocrine stress responses. Neuroimage. 2007;35(4):1601-12.

8. Hertenstein MJ, Keltner D, App B, Bulleit BA, Jaskolka AR. Touch communicates distinct emotions. Emotion. 2006;6(3):528-33.

9. Kirsch LP, Krahé C, Blom N, Crucianelli L, Moro V, Jenkinson PM, et al. Reading the mind in the touch: Neurophysiological specificity in the communication of emotions by touch. Neuropsychologia. 2017;

10. Von Mohr M, Kirsch LP, Fotopoulou A. The soothing function of touch: Affective touch reduces feelings of social exclusion. Sci Rep. 2017;7(1).

11. Harlow HF, Zimmerman RR. Affectional responses in the infant monkey. Science (80- ). 1959;130(3373):421-32.

12. Nelson EE, Panksepp J. Brain substrates of mother-infant attachment: Contributions of opoids, oxytocin and norepinephrine. Neurosci Biobehav Rev. 1998;22(3):437-52.

13. Sharp H, Pickles A, Meaney M, Marshall K, Tibu F, Hill J. Frequency of Infant Stroking Reported by Mothers Moderates the Effect of Prenatal Depression on Infant Behavioural and Physiological Outcomes. PLoS One. 2012;7(10). 
14. Maitre NL, Key AP, Chorna OD, Slaughter JC, Matusz PJ, Wallace MT, et al. The Dual Nature of Early-Life Experience on Somatosensory Processing in the Human Infant Brain. Curr Biol. 2017;27(7):1048-54.

15. Morrison I, Löken LS, Olausson H. The skin as a social organ. In: Experimental Brain Research. 2010. p. 305-14.

16. Burgoon JK, Walther JB, Baesler EJ. Interpretations, Evaluations, and Consequences of Interpersonal Touch. Hum Commun Res. 1992;19(2):237-63.

17. Fisher JD, Rytting M, Heslin R. Hands touching hands: Affective and evaluative effects of an interpersonal touch. Sociometry. 1976 Dec 1:416-21.

18. Hornik J. Tactile stimulation and consumer response. Journal of consumer research. 1992 Dec 1;19(3):449-58.

19. Crusco AH, Wetzel CG. The Midas Touch The Effects of Interpersonal touch on Restaurant Tipping. Personal Soc Psychol Bull. 1984;10(4):512-7.

20. Joule R-V, Guéguen N. Touch, Compliance, and Awareness of Tactile Contact. Percept Mot Skills [Internet]. 2007;104(2):581-8. Available from: http://journals.sagepub.com/doi/10.2466/pms.104.2.581-588

21. Dunbar R. Groups, gossip and the evolution of language. New Asp Hum Ethol. 1997;77-89.

22. Dunbar RIM. The social role of touch in humans and primates: Behavioural function and neurobiological mechanisms. Vol. 34, Neuroscience and Biobehavioral Reviews. 2010. p. 260-8.

23. Amico JA, Vollmer RR, Karam JR, Lee PR, Li X, Koenig Jl, et al. Centrally administered oxytocin elicits exaggerated grooming in oxytocin null mice. Pharmacol Biochem Behav. 2004;78(2):333-9.

24. Stack DM, Muir DW. Adult Tactile Stimulation during Face-to-Face Interactions Modulates Five-Month-Olds' Affect and Attention. Child Dev. 1992;63(6):1509-25.

25. Feldman $R$, Singer $M$, Zagoory $O$. Touch attenuates infants' physiological reactivity to stress. Dev Sci. 2010;13(2):271-8.

26. Bowlby J. Attachment and loss: Attachment. Vol. 1, Attachment. 1969. 326 p.

27. Ainsworth MS. Infant-mother attachment. Vol. 34, American psychologist. 1979. p. 932-7.

28. Weiss SJ, Wilson P, Hertenstein MJ, Campos R. The tactile context of a mother's caregiving: Implications for attachment of low birth weight infants. Infant Behav Dev. 2000;23(1):91111.

29. Beebe B, Jaffe J, Markese S, Buck K, Chen H, Cohen P, et al. The origins of 12-month attachment: A microanalysis of 4-month mother-infant interaction. Attach Hum Dev. 2010;12(1-2):3-141.

30. Nelson H, Geher G. Mutual grooming in human dyadic relationships: An ethological perspective. Vol. 26, Current Psychology. 2007. p. 121-40.

31. Korosi A, Baram TZ. Plasticity of the stress response early in life: Mechanisms and significance. Dev Psychobiol. 2010;52(7):661-70.

32. Nelson EE, Panksepp J. Brain substrates of infant-mother attachment: Contributions of opioids, oxytocin, and norepinephrine. Neurosci Biobehav Rev. 1998;22:437-52.

33. Ditzen B, Neumann ID, Bodenmann G, von Dawans B, Turner RA, Ehlert U, et al. Effects of different kinds of couple interaction on cortisol and heart rate responses to stress in women. Psychoneuroendocrinology. 2007;32(5):565-74.

34. Morrison I. Keep calm and cuddle on: social touch as a stress buffer. Adapt Hum Behav Physiol. 2016;(2):344-62.

35. Tai K, Zheng $X$, Narayanan J. Touching a teddy bear mitigates negative effects of social 
exclusion to increase prosocial behavior. Vol. 2, Social Psychological and Personality Science. 2011. p. 618-26.

36. Krahé C, Drabek MM, Paloyelis Y, Fotopoulou A. Affective touch and attachment style modulate pain: a laser-evoked potentials study. Philosophical Transactions of the Royal Society B: Biological Sciences. 2016 Nov 19;371(1708):20160009.

37. von Mohr M, Krahe $C$, Beck B, Fotopoulou A. The social buffering of pain by affective touch: A laser-evoked potential study in romantic couples. Soc Cogn Affect Neurosci. 2018;13(11):1121-30.

38. Beckes L, Coan JA. Social Baseline Theory: The Role of Social Proximity in Emotion and Economy of Action. Soc Personal Psychol Compass. 2011 Dec;5(12):976-88.

39. Coan JA. The social regulation of emotion. In: Decety J, Cacioppo JT, editors. The Oxford Handbook of Social Neuroscience. New York: Oxford University Press; 2011. p. 614-23.

40. Decety J, Fotopoulou A. Why empathy has a beneficial impact on others in medicine: unifying theories. Frontiers in behavioral neuroscience. 2015 Jan 14;8:457.

41. Atzil S, Barrett LF. Social regulation of allostasis: Commentary on "Mentalizing homeostasis: The social origins of interoceptive inference" by Fotopoulou and Tsakiris. Neuropsychoanalysis. 2017 Jan 2;19(1):29-33.

42. Fotopoulou A, Tsakiris M. Mentalizing homeostasis: The social origins of interoceptive inference-replies to Commentaries. Neuropsychoanalysis. 2017;19(1):71-6.

43. Rajkumar RP. COVID-19 and mental health: A review of the existing literature. Asian J Psychiatr. 2020;52.

44. Debrot A, Schoebi D, Perrez M, Horn AB. Touch as an interpersonal emotion regulation process in couples' daily lives: The mediating role of psychological intimacy. Personality and Social Psychology Bulletin. 2013 Oct;39(10):1373-85.

45. Chester DS, DeWall CN, Pond RS. The push of social pain: Does rejection's sting motivate subsequent social reconnection? Cogn Affect Behav Neurosci. 2016;16(3):541-50.

46. Maner JK, DeWall CN, Baumeister RF, Schaller M. Does social exclusion motivate interpersonal reconnection? Resolving the "porcupine problem". J Pers Soc Psychol. 2007;92:42-55.

47. von Mohr M, Fotopoulou A. The cutaneous borders of interoception: active and social inference on pain and pleasure on the skin. In: Tsakiris $\mathrm{M}$, De Preester $\mathrm{H}$, editors. The interoceptive mind: from homeostasis to awareness. 2018.

48. Wei M, Russell DW, Mallinckrodt B, Vogel DL. The Experiences in Close Relationship Scale (ECR)-short form: Reliability, validity, and factor structure. Journal of personality assessment. 2007 Mar 27;88(2):187-204.

49. Krahé C, von Mohr M, Gentsch A, Guy L, Vari C, Nolte T, et al. Sensitivity to Affective Touch Depends on Adult Attachment Style. bioRxiv. 2018.

50. Trotter PD, McGlone F, Reniers RLEP, Deakin JFW. Construction and Validation of the Touch Experiences and Attitudes Questionnaire (TEAQ): A Self-report Measure to Determine Attitudes Toward and Experiences of Positive Touch. J Nonverbal Behav. 2018;42(4):379416.

51. Stack DM, Muir DW. Tactile stimulation as a component of social interchange: New interpretations for the still-face effect. British Journal of Developmental Psychology. 1990 Jun;8(2):131-45.

52. Field T. Touch for socioemotional and physical well-being: A review. Vol. 30, Developmental Review. 2010. p. 367-83.

53. Russell D, Peplau LA, Cutrona CE. The revised UCLA Loneliness Scale: Concurrent and 
discriminant validity evidence. J Pers Soc Psychol. 1980;39(3):472-80.

54. Marteau TM, Bekker H. The development of a six-item short-form of the state scale of the Spielberger State-Trait Anxiety Inventory (STAI). Br J Clin Psychol. 1992;31(3):301-6.

55. Tabachnick BG, Fidell LS, Ullman JB. Using multivariate statistics. Boston, MA: Pearson; 2007 Mar 3.

56. Suvilehto JT, Glerean E, Dunbar RI, Hari R, Nummenmaa L. Topography of social touching depends on emotional bonds between humans. Proceedings of the National Academy of Sciences. 2015 Nov 10;112(45):13811-6.

57. Beßler R, Bendas J, Sailer U, Croy I. The "Longing for Interpersonal Touch Picture Questionnaire": Development of a new measurement for touch perception. Int J Psychol. 2020;55(3):446-55.

58. Maner JK, DeWall CN, Baumeister RF, Schaller M. Does social exclusion motivate interpersonal reconnection? Resolving the "porcupine problem." J Pers Soc Psychol. 2007;92(1):42-55.

59. Ellingsen DM, Leknes S, Løseth G, Wessberg J, Olausson H. The neurobiology shaping affective touch: Expectation, motivation, and meaning in the multisensory context. Vol. 6, Frontiers in Psychology. 2016.

60. Hazen C, Shaver P. Romantic love conceptualized as an attachment process. Journal of personality and social psychology. 1987;52(3):511-24.

61. Bartholomew K, Horowitz LM. Attachment styles among young adults: a test of a fourcategory model. Journal of personality and social psychology. 1991 Aug;61(2):226.

62. Ravitz P, Maunder R, Hunter J, Sthankiya B, Lancee W. Adult attachment measures: A 25year review. Vol. 69, Journal of Psychosomatic Research. 2010. p. 419-32.

63. Krahé C, Paloyelis Y, Condon H, Jenkinson PM, Williams SCR, Fotopoulou A. Attachment style moderates partner presence effects on pain: A laser-evoked potentials study. Soc Cogn Affect Neurosci. 2015;10(8):1030-7.

64. Sailer U, Ackerley R. Exposure shapes the perception of affective touch. Developmental Cognitive Neuroscience. 2018;

65. Waters E, Merrick S, Treboux D, Crowell J, Albersheim L. Attachment security in infancy and early adulthood: A twenty-year longitudinal study. Child development. 2000 May;71(3):6849. 


\section{Supplementary Materials}

Table S1. Model results for effects of days practicing social distancing on wanting touch

Effect Level $\quad b \quad$ SE $p$-value Confidence Intervals
Lower Upper

Type of touch

$\begin{array}{llllll}\text { Friendly } & -26.76 & .98 & .000 & -28.66 & -24.86 \\ \text { Professional } & -50.23 & .92 & .000 & -52.04 & -48.44\end{array}$

Days in lockdown

$\begin{array}{llll}.20 & .08 & .009 & .05\end{array}$

.35

Type of touch $\mathrm{x}$

Days in lockdown

$\begin{array}{llllll}\text { Friendly } & -.24 & .08 & .004 & -.40 & -.07 \\ \text { Professional } & -.34 & .08 & .000 & -.50 & -.18\end{array}$

c. Lack of touch

$63.68 \quad .86 \quad .000$

62.01

65.36

Note. Intimate touch was the base level

Table S2. Model results for effects of adult attachment on wanting touch

Effect Level $\quad b \quad$ SE $p$-value Confidence Intervals

Lower Upper

Type of touch

$\begin{array}{llllll}\text { Friendly } & -27.51 & 1.20 & .000 & -29.88 & -25.14 \\ \text { Professional } & -50.43 & 1.14 & .000 & -52.68 & -48.19\end{array}$

Attachment

$3.07 \quad .86 \quad .000$

1.38

4.76

anxiety

Type of touch $x$

Attachment

anxiety

$\begin{array}{lccccc}\text { Friendly } & 3.13 & .96 & .001 & 1.25 & 5.01 \\ \text { Professional } & -.96 & .96 & .315 & -2.83 & .91 \\ & & & & & \\ & -13.61 & .94 & .000 & -15.45 & -11.76\end{array}$

Attachment

avoidance

Type of touch $x$

Attachment

avoidance

$\begin{array}{lccccc}\text { Friendly } & 8.76 & 1.05 & .000 & 6.70 & 10.81 \\ \text { Professional } & 11.71 & 1.04 & .000 & 9.67 & 13.76\end{array}$


Avoidance $x$

$2.15 \quad .75 \quad .004$

.69

3.61

anxiety

Type of touch $\mathrm{x}$

avoidance $x$

anxiety

$\begin{array}{lccccc}\text { Friendly } & -.75 & .83 & . .365 & -2.38 & .87 \\ \text { Professional } & -1.10 & .83 & .185 & -2.72 & .53\end{array}$

$\begin{array}{llllll}\text { c. Lack of touch } & .25 & .02 & .000 & .21 & .28\end{array}$

Note. Intimate touch was the base level

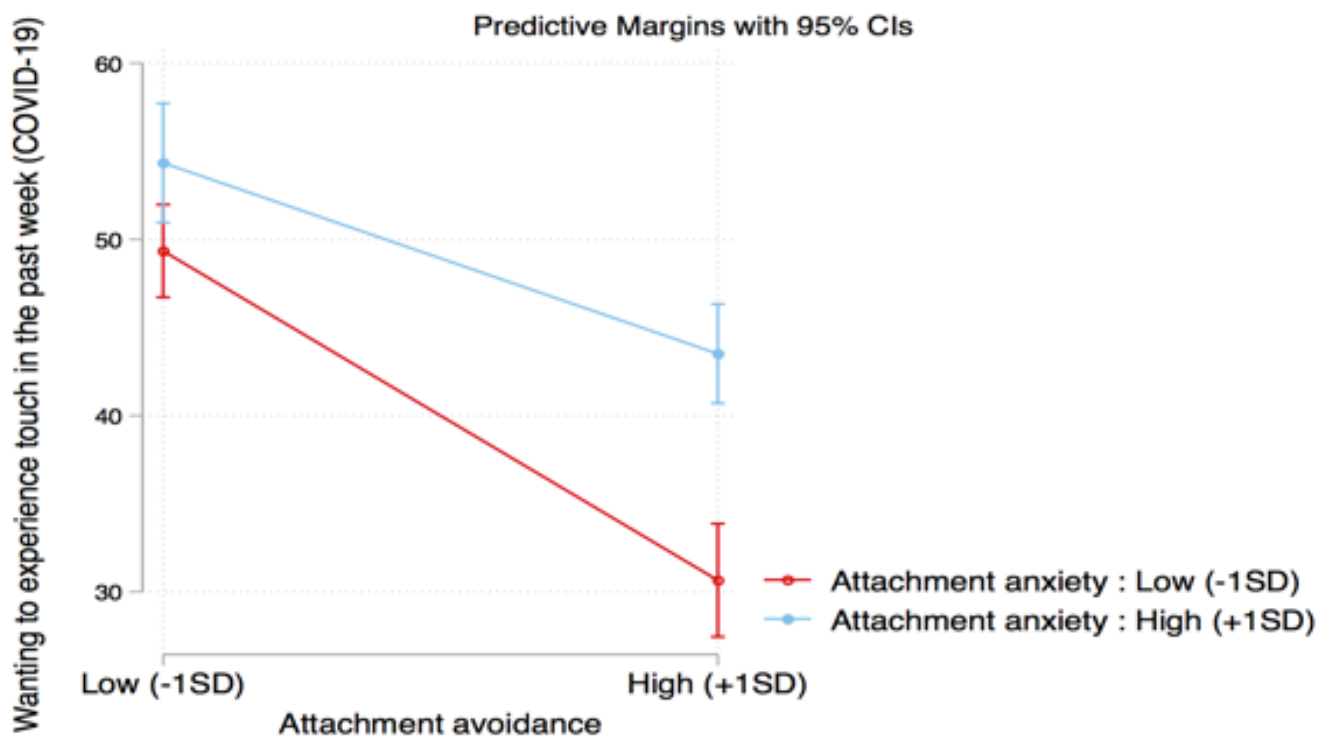

Figure S1. Effects of Attachment avoidance by Attachment anxiety interaction on wanting touch plotted at at low (-1 s.d.) and high (+1 s.d.) attachment anxiety and attachment avoidance scores. Error bars denote SE of the predicted margins. Follow-up tests indicate that at lower values of anxious and avoidance scores, the more the wanting to experience touch, $p^{\prime} s<.004$; however, under our predetermined alpha level of 0.01 , there was no significant difference in wanting touch between low values of anxious and avoidant scores and high anxiety and low avoidance, p=.019.

Table S3. Model results for effects of touch attitudes and experiences on wanting touch

\begin{tabular}{llllll}
\hline Effect & Level & $b$ & SE & p-value & $\begin{array}{c}\text { Confidence Intervals } \\
\text { Lower }\end{array}$ \\
\hline
\end{tabular}

Type of touch 


$\begin{array}{llllll}\text { Friendly } & -26.04 & .96 & .000 & -27.93 & -24.16 \\ \text { Professional } & -49.76 & .91 & .000 & -51.55 & -47.98\end{array}$

Touch attitudes

$\begin{array}{lll}2.08 & .15 & 000\end{array}$

1.79

2.37

and experiences

Type of touch $x$

touch attitudes

and experiences

$\begin{array}{lccccc}\text { Friendly } & -.19 & .17 & .243 & -.52 & -.13 \\ \text { Professional } & -1.13 & .17 & .000 & -1.45 & -.80\end{array}$

c. Lack of touch

.2

$.82 \quad .000$

62.51

65.75

Note. Intimate touch was the base level

Table S4. Model results for effects of touch attitudes and experiences items on wanting touch

\begin{tabular}{|c|c|c|c|c|c|}
\hline \multirow[t]{2}{*}{ Effect } & \multirow[t]{2}{*}{$b$} & \multirow[t]{2}{*}{ SE } & \multirow[t]{2}{*}{$p$-value } & \multicolumn{2}{|c|}{ Confidence Intervals } \\
\hline & & & & Lower & Upper \\
\hline TEAQ_bath & 1.52 & .43 & .000 & .67 & 2.36 \\
\hline TEAQ_parents & .44 & .52 & .397 & -.58 & 1.45 \\
\hline TEAQ_tuckparents & .69 & .53 & .195 & -.35 & 1.74 \\
\hline TEAQ_huggreet & 3.35 & .51 & .000 & 2.35 & 4.36 \\
\hline TEAQ_skin & 7.08 & .64 & .000 & 5.83 & 8.33 \\
\hline TEAQ_gettinghug & 2.05 & .43 & .000 & 1.20 & 2.90 \\
\hline TEAQ stranger & -1.65 & .47 & .000 & -2.58 & -.72 \\
\hline
\end{tabular}

Note. Intimate touch was the base level. We observe the same pattern of results when controlling for lack of touch.

Table S5. Model results for effects of psychiatric condition on lacking touch. Psychiatric condition was entered as a categorical predictor

\begin{tabular}{lccccc}
\hline Effect & $b$ & SE & p-value & \multicolumn{2}{c}{ Confidence Intervals } \\
& & & & Lower & Upper \\
\hline & & & & & \\
Anxiety & -2.96 & 3.35 & .377 & -9.53 & 3.61 \\
Depression & -2.18 & 3.32 & .511 & -8.67 & 4.32 \\
Anxiety and depression & -8.21 & 3.83 & .032 & -15.72 & -.70 \\
Other & -11.45 & 2.42 & .000 & -16.20 & -6.71
\end{tabular}

Note. Non-psychiatric condition was the base level. We observe the same pattern Note. Nonpsychiatric condition was the base level. 


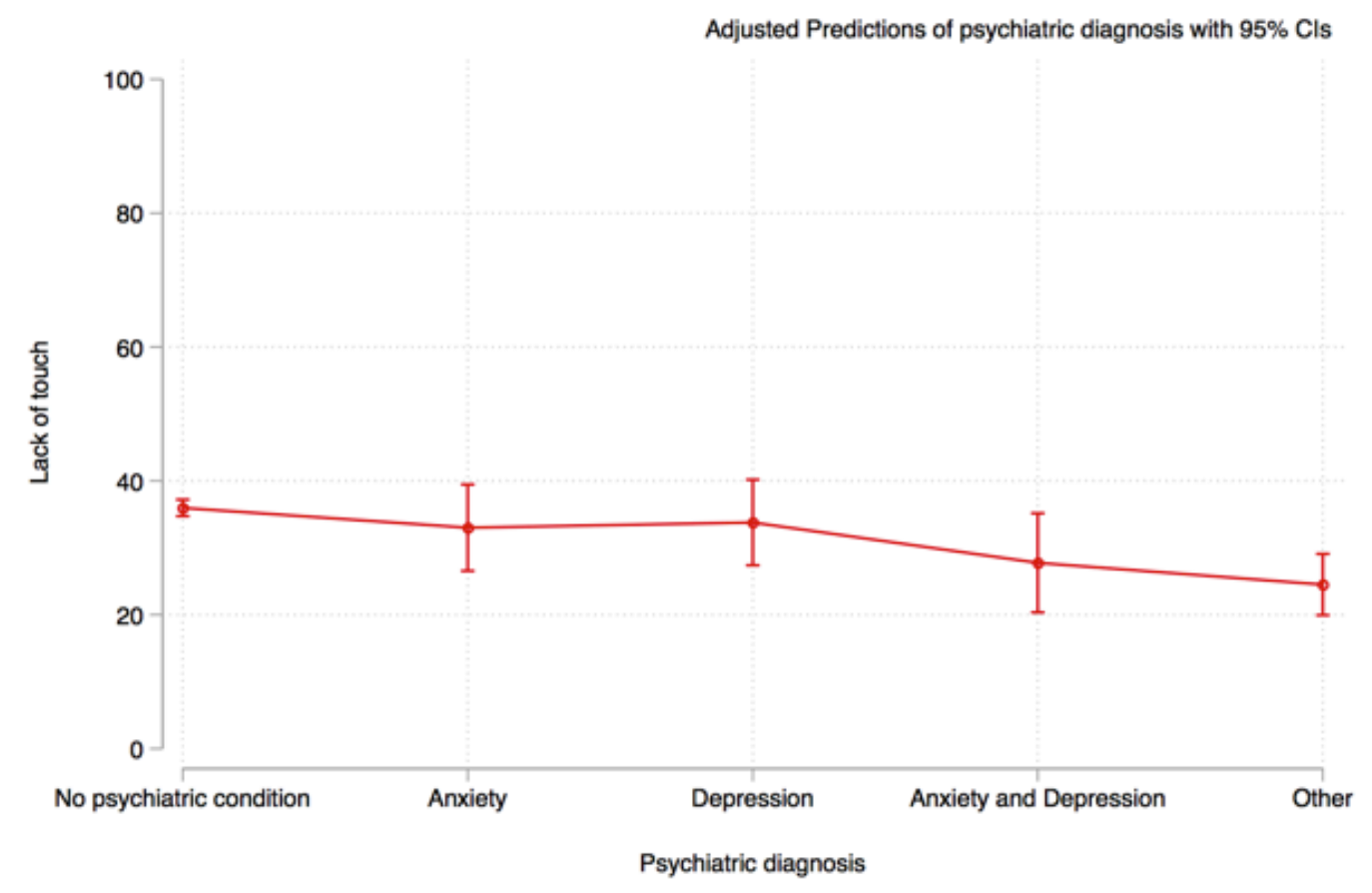

Figure S2. Effects of psychiatric diagnosis on lack of touch.

Table S6. Model results for effects of psychiatric condition on wanting touch. Psychiatric condition was entered as a categorical predictor

\begin{tabular}{lccccc}
\hline Effect & $b$ & SE & $p$-value & \multicolumn{2}{c}{ Confidence Intervals } \\
& & & & Lower & Upper \\
\hline & & & & & \\
Anxiety & -.61 & 3.62 & .867 & -7.69 & 6.49 \\
Depression & 3.73 & 3.59 & .297 & -3.29 & 10.77 \\
Anxiety and depression & 1.11 & 4.14 & .788 & -6.99 & 9.22 \\
Other & -2.09 & 2.62 & .423 & -7.22 & 3.03 \\
& & & & &
\end{tabular}

Note. Non-psychiatric condition was the base level. We observe the same pattern of results when controlling for lack of touch 
Figure S3. Spearman correlations between variables of interest. A. Correlations between average lack of touch, average wanting touch and variables of interests. B. Correlations between the different type of touch lacked and wanted, and variables of interests. Spearman rho are represented in each cell, with blue for positive correlations and red for negative correlations. Crossed cell represent non-significant correlations ( $p>0.01$ ).

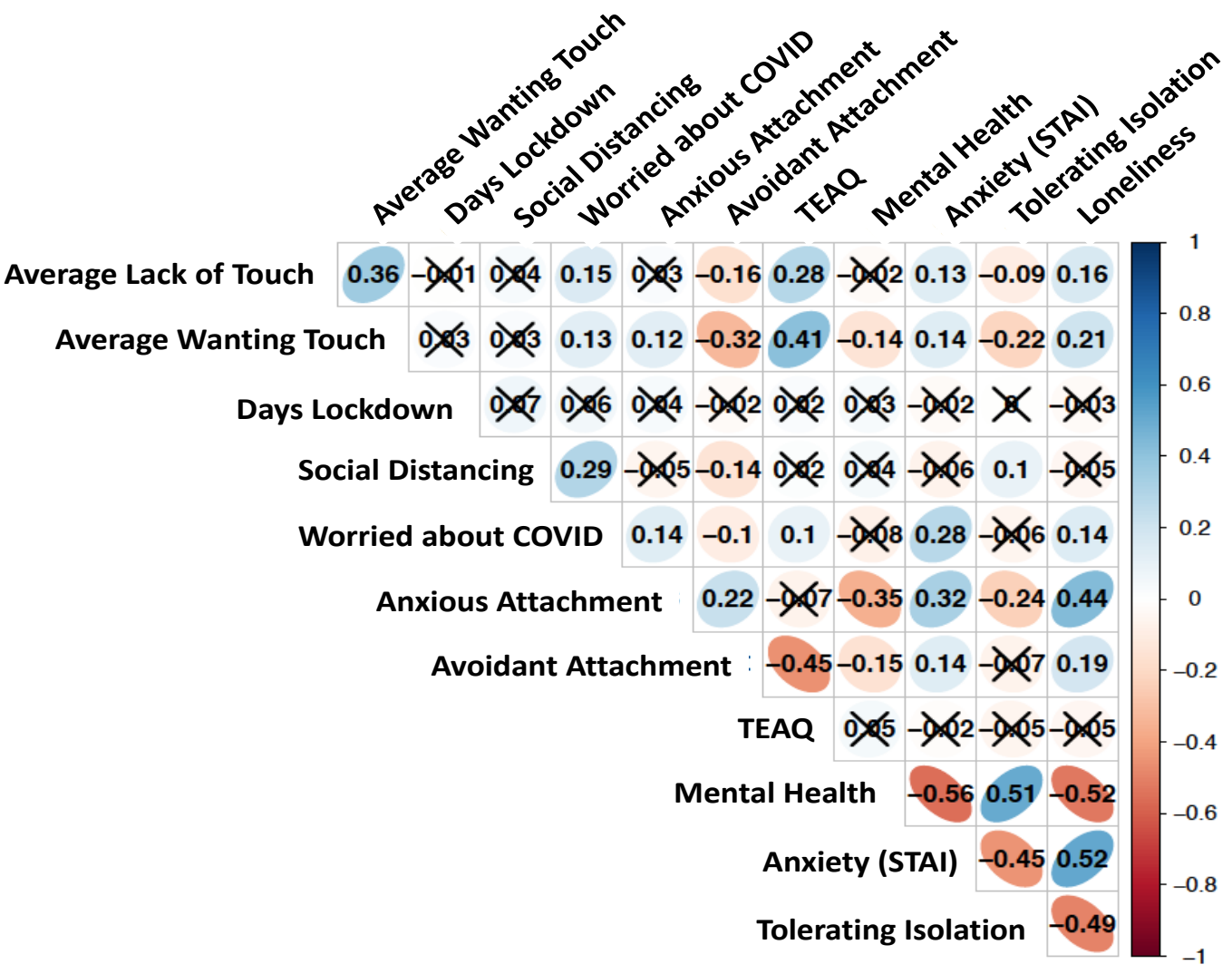

B.

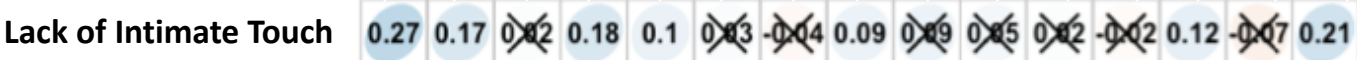

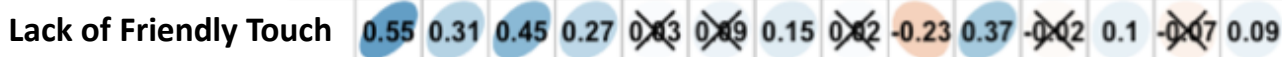

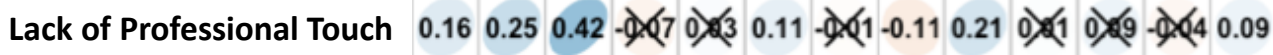
Wanting Intimate Touch 0.480 .240$)(50)(6 \quad 0.090)(1-0.450 .36-1)(60)(2-2)(60)(2$

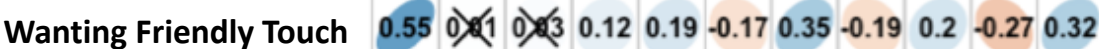

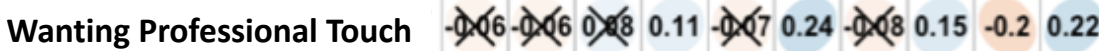

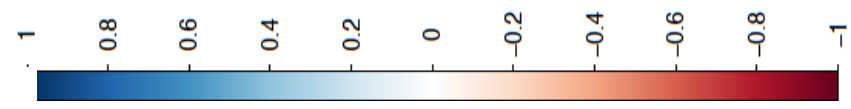


